Prospective Pilot Study

\title{
e Ultrasound Guided Intercostobrachial Nerve Blockade in Patients with Persistent Pain after Breast Cancer Surgery: A Pilot Study
}

Nelun Wijayasinghe, MBBS, FRCA, Helle M. Duriaud, RN, Henrik Kehlet, MD, PhD, DMSc, and Kenneth G. Andersen, MD, PhD

From: Section for Surgical Pathophysiology, Rigshospitalet, University of Copenhagen,

Copenhagen, Denmark

Dr Wijayasinghe is an anesthesiologist and clinical researcher. RN Duriaud is a research nurse. Professor Kehlet is a surgeon and the clinical professor of surgical pathophysiology. Dr. Andersen is a clinical researcher.

Address Correspondence: Dr Nelun Wijayasinghe Section for Surgical Pathophysiology 4074 Rigshospitalet

University of Copenhagen, Blegdamsvej 9 DK-2100 Copenhagen, Denmark E-mail: nelunwij@gmail.com

Disclaimer:This study was funded by a grant from the Danish Cancer Society and the study is part of the Europain Collaboration, which has received support from the Innovative Medicines Initiative Joint Undertaking, under grant agreement no. 115007 resources which are composed of financial contributions from the European Union's Seventh Framework Programme (FP7/2007D2013) and EFPIA companies in kind contribution. This study was also sponsored by an ESRA grant.

The Simon Fougner Hartmanns Family Foundation kindly provided the Venue 40, GE Healthcare ultrasound unit. They had no influence or interference after the protocol was designed.

Manuscript received: 06-13-2015 Revised manuscript received: 08-05-2015 Accepted for publication: 08-07-2015

Free full manuscript: www.painphysicianjournal.com
Background: Persistent pain after breast cancer surgery (PPBCS) affects $25-60 \%$ of breast cancer survivors and damage to the intercostobrachial nerve (ICBN) has been implicated as the cause of this predominantly neuropathic pain. Local anesthetic blockade of the ICBN could provide clues to pathophysiological mechanisms as well as aiding diagnosis and treatment of PPBCS but has never been attempted.

Objectives: To assess the feasibility of ICBN blockade and assess its effects on pain and sensory function in patients with PPBCS.

Study Design: This prospective pilot study was performed in 2 parts: Part 1 determined the sonoanatomy of the ICBN and part 2 examined effects of the ultrasound-guided ICBN blockade in patients with PPBCS.

Setting: Section for Surgical Pathophysiology at Rigshospitalet, Copenhagen, Denmark.

Methods: Part 1: Sixteen unoperated, pain free breast cancer patients underwent systematic ultrasonography to establish the sonoanatomy of the ICBN. Part 2: Six patients with PPBCS who had pain in the axilla and upper arm were recruited for the study. Summed pain intensity (SPI) scores and sensory function were measured before and 30 minutes after the block was administered. SPI is a combined pain score of numerical rating scale (NRS) at rest, movement, and $100 \mathrm{kPa}$ pressure applied to the maximum point of pain using pressure algometry ( $\mathrm{max}$ = 30). Sensory function was measured using quantitative sensory testing, which consisted of sensory mapping, thermal thresholds, suprathreshold heat pain perception as well as heat and pressure pain thresholds. The ICBN block was performed under ultrasound guidance and $10 \mathrm{~mL}$ $0.5 \%$ bupivacaine was injected.

Outcome Assessment: The ability to perform the ICBN block and its analgesic and sensory effects.

Results: Only the second intercostal space could be seen on ultrasound which was adequate to perform the ICBN block. The mean difference in SPI was -9 NRS points $(95 \% \mathrm{Cl}:-14.1$ to -3.9$)$, $P=0.006$. All patients had pre-existing areas of hypoesthesia which decreased in size in $4 / 6$ patients after the block.

Limitations: The main limitation of this pilot study is its small sample size, but despite this, a statistically significant effect was observed.

Conclusion: We have successfully managed to block the ICBN using ultrasound guidance and demonstrated an analgesic effect in patients in PPBCS calling for placebo-controlled studies.

Key words: Anesthesia, local; intercostobrachial nerve; mastectomy; nerve block; neuralgia

Pain Physician 2016; 19:E309-E317 
P ersistent pain after breast cancer surgery (PPBCS) is predominantly a neuropathic pain syndrome affecting $25-60 \%$ of patients treated for breast cancer $(1,2)$ and may develop years after surgery $(3)$. Patients experience pain in the axilla, lateral chest wall, and upper arm, accompanied by sensory disturbances in the territory supplied by the intercostobrachial nerve (ICBN) (4). The ICBN arises from the lateral cutaneous branch of the second intercostal nerve. The nerve pierces the external intercostal muscle and serratus anterior then crosses the axilla to the medial side of the arm and supplies the upper half of the medial and posterior part of the arm (5). The nerve's path from the anterior chest wall through the axilla makes it vulnerable to damage during all forms of breast cancer surgery especially axillary lymph node dissection (6). However strategies to preserve the ICBN and prevent the development of PPBCS have been equivocal and have failed to prevent the problem $(1,4)$.

It has been suggested that ICBN blockade could be potentially useful in understanding the pathophysiology and aiding diagnosis of PPBCS (7), yet this has never been described. Studies of peripheral nerve blockade in other neuropathic pain syndromes have demonstrated an analgesic effect that also outlasts the conduction block of the local anesthetic suggesting potential use of nerve blocks as treatment (8). Therefore, the aim of this pilot study was to assess the feasibility of an ultrasound guided ICBN block using local anesthetic and to evaluate its effect(s) on pain and sensory function in PPBCS.

\section{Methods}

The study was approved by the ethics committee of the Capital Region of Denmark (H-3-2013-172) and registered in ClinicalTrials.gov (NCT02336529). The study was prospective and conducted in 2 parts: Part 1 determined the sonoanatomy of the ICBN in unoperated, pain free breast cancer patients and Part 2 assessed effects of the local anesthetic blockade on pain and sensory function in patients with PPBCS. All parts of the study were carried out at Rigshospitalet, Copenhagen, Denmark, from April to October 2014.

\section{Part 1}

Informed, verbal consent was taken from 16 patients awaiting breast cancer surgery. Inclusion criteria were women with a diagnosis of breast cancer scheduled for any type of breast cancer surgery and aged $\geq$ 18 years. Exclusion criteria were women with previous surgery to the axilla on the same side as their breast cancer and those unable to give consent for the procedure. Prior to scanning, anatomical descriptions of the ICBN and radiology experts in ultrasonography were consulted to assess the likelihood of finding the ICBN in the anterolateral chest wall. It was concluded that the ICBN was too small to be seen with ultrasound. However, previous anatomical studies have shown the nerve to have a reliable course in the second intercostal space (IC2) in the chest wall (5). Therefore, the aim of scanning was to identify the IC2. A list of the anatomical landmarks was made for use as a checklist while scanning. The side to be operated was scanned using the same ultrasound machine (Venue 40, GE Healthcare, Waukesha, WI, USA) using a $12 \mathrm{GHz}$ linear probe and the same operator (NW). A series of 3 ultrasound pictures were taken for each patient in order to describe the sonoanatomy of the ICBN.

\section{Part 2}

Patients were recruited from a cohort of patients that had participated in previous studies $(3,9,10)$ where they had filled out questionnaires concerning pain after breast cancer surgery. Four hundred and twenty-six patients were identified as potential suitable candidates. Inclusion criteria were women aged $\geq 18$ years that had had breast cancer surgery, developed pain in the axilla, upper arm, or lateral chest wall $>6$ months after surgery and a pain score $\geq 4$ on the numerical rating scale (NRS) on a daily basis. Exclusion criteria were patients with bilateral breast cancer (with bilateral interventions), previous cosmetic breast surgery, $<12$ months since the last intervention for breast cancer, pregnant or breast feeding, $\mathrm{BMI}>40 \mathrm{~kg} \mathrm{~m}^{-2}$, neurological disease, drug or alcohol abuse, psychiatric disease, inability to understand Danish and mentally incapacitated. Further screening was undertaken via telephone or in person to verify that the pain was likely to be due solely to ICBN damage. Written informed consent was taken from all patients. Patients were scheduled for one visit where the following observations were determined before and after the administration of the ultrasound guided ICBN block: summed pain intensity score (SPI), range of shoulder movement, scapula symmetry, and quantitative sensory testing (QST).

\section{Pain Scores}

The SPI is a combination of 3 pain scores measured using NRS. Thus, SPI = NRSr + NRSm + NRSp where NRSr is pain at rest, NRSm is pain on movement (arm abduction), and NRSp is pain with $100 \mathrm{kPa}$ pressure applied to 
the point of maximum pain (Pmax) (11). NRSp was assessed using a pressure algometer (Somedic AB, Hörby, Sweden) consisting of a $1 \mathrm{~cm}^{2}$ felt tipped probe, applied perpendicularly to the skin with a rate of $20-30 \mathrm{kPa} / \mathrm{s}$.

\section{Quantitative Sensory Testing (QST)}

QST consisted of sensory mapping, warmth detection thresholds (WDT), cool detection thresholds (CDT), heat pain thresholds (HPT), supra-threshold heat pain response (STH), and pressure pain thresholds (PPT) according to our standardized protocol (10). Sensory mapping was performed in the axilla, arm, anterior, lateral, and posterior chest wall with a brush, warm metal roller $\left(40^{\circ} \mathrm{C}\right.$ ), and cool metal roller $\left(25^{\circ} \mathrm{C}\right)$ (Somedic $A B$, Sweden) delineating areas with warm and cool hypo/hyperaesthesia or allodynia. These areas were marked with a felt tipped pen on the skin and photographed with a ruler. The area of each sensory map was calculated using graphical software (Canvas 8.0.3 Deneba, ACD Systems International, Victoria, Canada). Thermal thresholds were determined using a computerized thermode (active surface $2.5 \times 5 \mathrm{~cm}$; MSA, Somedic AB, Sweden) placed over the Pmax. Three thresholds were obtained for each stimulus and cut-off temperatures were $52^{\circ} \mathrm{C}$ for heat stimuli and $5^{\circ} \mathrm{C}$ for cold stimuli to avoid tissue damage. The supra-threshold heat pain response (STH) was obtained by heating the thermode to $47^{\circ} \mathrm{C}$ and maintaining this temperature for 5 seconds while still on the skin. After the 5 seconds, the thermode was removed and the patient was asked to rate their pain using the NRS. Pressure pain thresholds (PPT) were determined using pressure algometry (see above) with an upper limit of $350 \mathrm{kPa}$.

\section{Longer Term Effects}

Patients filled out a pain diary for 5 consecutive days prior to their visit and a questionnaire on painrelated functional impairment the day before the visit. For the pain diary, the patients noted their pain scores using the NRS twice a day in 3 predetermined states: at rest (supine), on movement $\left(90^{\circ}\right.$ arm abduction), and with manual pressure on the Pmax. Sleep quality was also assessed in the pain diary using a numerical scoring system from 0 to 10 where $0=$ pain had no effect on sleep, $10=$ pain totally affected sleep. The pain-related functional impairment questionnaire was a standardized, procedure specific, validated questionnaire that has been used in previous studies which measures reduced physical function due to pain in the area of surgery on a $0-100$ scale (12). The morning after their visit, the patients filled out another pain diary for 7 consecutive days and one week after their visit they completed another functional questionnaire.

\section{Statistical Analyses}

This pilot study was primarily a descriptive study of the ICBN block. However, our secondary outcome was to calculate the effect, i.e., mean difference, in SPI after the ICBN block. Paired t-tests comparing preblock values with post-block values were performed on the SPI data as well as on the individual pain scores (NRSr, NRSm, NRSp). The secondary outcome was also the QST data, where t-tests comparing pre-block to post-block values were also performed. All secondary outcome data are presented as mean differences $(95 \%$ $\mathrm{Cl} ; P$ value). All analyses were performed using statistical software (MedCalc 14.8.1, MedCalc Software bvba, Ostend, Belgium). A sample size calculation was not done as this is a pilot study and no previous data were available in the literature. However, a sample size calculation was performed using the results of the present study for a future randomized controlled trial (RCT) and to determine the context of this study (see results).

\section{Results}

\section{Part 1}

All 16 patients awaiting breast cancer surgery agreed to be scanned. Their mean (SD) age was 57 (10.5) years and median (IQR) BMI was 24.9 (24.0 to 31.3) $\mathrm{kg} \mathrm{m}^{-2}$.

The ultrasound probe was placed in the parasagittal plane in the most lateral part of the clavicle with the probe at a right angle to the clavicle. The first rib was identified by scanning over the clavicle and looking for the axillary vein and artery situated above the first rib, i.e., medial to the coracoid process (Fig. 1A). The probe was moved inferiorly to find the second rib (Fig.1B). The probe was then moved more laterally (keeping the second rib in view at all times) and given an oblique angle so that the inferior edge of the probe was on the anterior axillary line in order to visualize the serratus anterior. Once the serratus anterior was seen, the probe was moved inferiorly so that the third rib came into view, i.e., the whole IC2 could be seen (Fig. 1C). The picture was taken when all of the following structures were identified in the same ultrasound picture: second and third rib, pectoralis major, pectoralis minor, serratus anterior, and pleura (Fig. 1C). All structures were visualized by ultrasound in 15/16 patients. The 


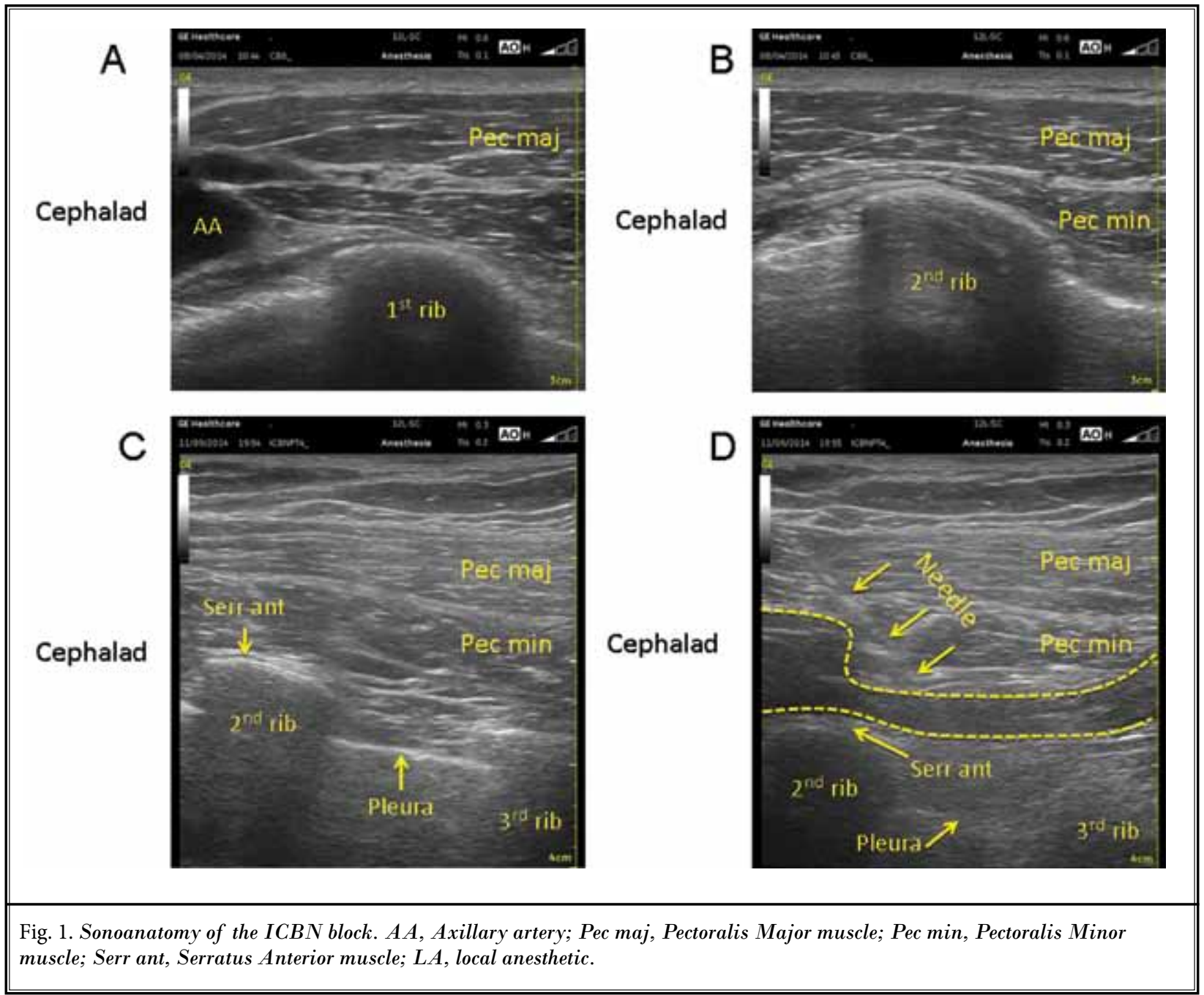

second rib could not be visualized in patient 8 with a $\mathrm{BMI}$ of $49 \mathrm{~kg} \mathrm{~m}^{-2}$, as only the lower border of pectoralis minor could be seen using the maximum depth $(6 \mathrm{~cm})$ available on our ultrasound apparatus.

\section{Part 2}

Ninety-two patients responded to the telephone and written invitations. Only 10/92 patients were eligible for enrollment into the study but 2 of those patients had insufficient pain on the visit day and 2 withdrew prior to the visit, therefore they were excluded. Thus, 6 patients with persistent neuropathic pain in the area supplied by ICBN were enrolled into the study, from July to October 2014 (Table 1).

\section{ICBN Block}

All blocks were performed by the same anesthesi- ologist (NW) using the same ultrasound unit (see Part 1) under aseptic conditions. As determined in part 1 of the study, IC2 was identified and a 22G block needle (Ultraplex®, Braun, Melsungen, Germany) was inserted under the skin using an in-plane technique (Fig. 1D). Our endpoint was the inferior edge of the second rib above the serratus anterior but under the pectoralis minor. Color Doppler ultrasonography was used to identify blood vessels in order to avoid intravascular injection and the pleura was always visualized. Ten $\mathrm{mL} 0.5 \%$ bupivacaine was injected and the spread of the injectate was visualized on the ultrasound screen (Fig. 1D). There were no immediate complications from the block.

\section{Pain Scores}

Fig. 2 shows the SPI (NRS) before and after the ICBN block: the mean difference was $-9.0(-14.1$ to $-3.9 ; P=$ 
Table 1. Patients' characteristics for those who received the ICBN block.

\begin{tabular}{|l|c|c|c|c|c|c|c|c||}
\hline \hline Patient & $\begin{array}{c}\text { Age } \\
(\mathbf{y r})\end{array}$ & $\begin{array}{c}\text { BMI } \\
\left(\mathbf{k g ~ m}^{-2}\right)\end{array}$ & $\begin{array}{c}\text { Type of breast } \\
\text { surgery }\end{array}$ & $\begin{array}{c}\text { Type of axillary } \\
\text { surgery }\end{array}$ & $\begin{array}{c}\text { Time since } \\
\text { surgery (yr) }\end{array}$ & $\begin{array}{c}\text { Duration of } \\
\text { pain (yr) }\end{array}$ & ChemoRx & RadioRx \\
\hline 1 & 70 & 23.0 & BCS & ALND & 7 & 7 & $\mathrm{Y}$ & $\mathrm{Y}$ \\
\hline 2 & 39 & 25.1 & BCS & SLN & 8 & 4 & $\mathrm{Y}$ & $\mathrm{Y}$ \\
\hline 3 & 72 & 22.0 & BCS & SLN & 4 & 2 & $\mathrm{~N}$ & $\mathrm{Y}$ \\
\hline 4 & 49 & 22.5 & MXT & SLN & 6 & 6 & $\mathrm{Y}$ & $\mathrm{Y}$ \\
\hline 5 & 52 & 22.3 & MXT & SLN & 1 & 1 & $\mathrm{~N}$ & $\mathrm{~N}$ \\
\hline 6 & 71 & 25.1 & MXT & ALND & 6 & 6 & $\mathrm{Y}$ & $\mathrm{Y}$ \\
\hline
\end{tabular}

BCS, breast conserving surgery; MXT, mastectomy; ALND, axillary lymph node dissection; SLN, sentinel lymph node biopsy; ChemoRx, chemotherapy; RadioRx, radiotherapy.

0.006). There was very little change in pain at rest, but there were reductions in pain on movement and with pressure: $\mathrm{NRSr}=-1.3(-3.0$ to $0.4 ; P=0.1), \mathrm{NRSm}=-3.9$ $(-7.0$ to $-0.8, P=0.02)$, and NRSp $=-3.8(-6.9$ to $-0.7 ; P$ $=0.03$ ).

\section{QST}

All patients exhibited preoperative areas of hypoesthesia to the brush and warm and cool rollers (Fig. $3 A$ ), which decreased in size in $4 / 6$ patients after the block (see Fig. 3B). Mean differences in hypoesthesia areas were $3.5 \mathrm{~cm}^{2}(-237.8$ to $244.9 ; P=0.97)$ for brush, $-209.7 \mathrm{~cm}^{2}(-757.5$ to $338.1 ; P=0.37)$ for warm, and $-261.1 \mathrm{~cm}^{2}(-720.7$ to $198.4 ; P=0.20)$ for cool.

There were no differences in all thermal detection thresholds and supra-thermal heat pain perception after the block: WDT $=-1.2^{\circ} \mathrm{C}(-3.98$ to $1.58 ; P=0.317)$, $\mathrm{CDT}=0.88^{\circ} \mathrm{C}(-1.85$ to $3.61 ; P=0.444), \mathrm{HPT}=-0.27^{\circ} \mathrm{C}$ (-4.22 to 3.68; $P=0.869), \mathrm{STH}=0.33(-0.75$ to $1.42 ; P=$ $0.465)$. However, there was an increase in PPT: $72.2 \mathrm{kPa}$ (2.3 to $142.0 ; P=0.045$ ).

\section{Longer Term Effects}

There were no complications reported a week after the block. Fig. 4 shows the results from the pain diaries before and after the block. One week after the block was administered 2 patients demonstrated an improvement in their functional impairment scores (-17 and -29). However, 3 patients experienced a slight increase $(13,4$, and 6$)$ and one patient had no change.

\section{Sample Size Calculation}

A sample size calculation was performed ( $G$ * power v3, Dusseldorf, Germany) using the results obtained in the present study for a future RCT where patients are matched and allocated to receive active treatment (block) or placebo (saline) and using the mean differ-

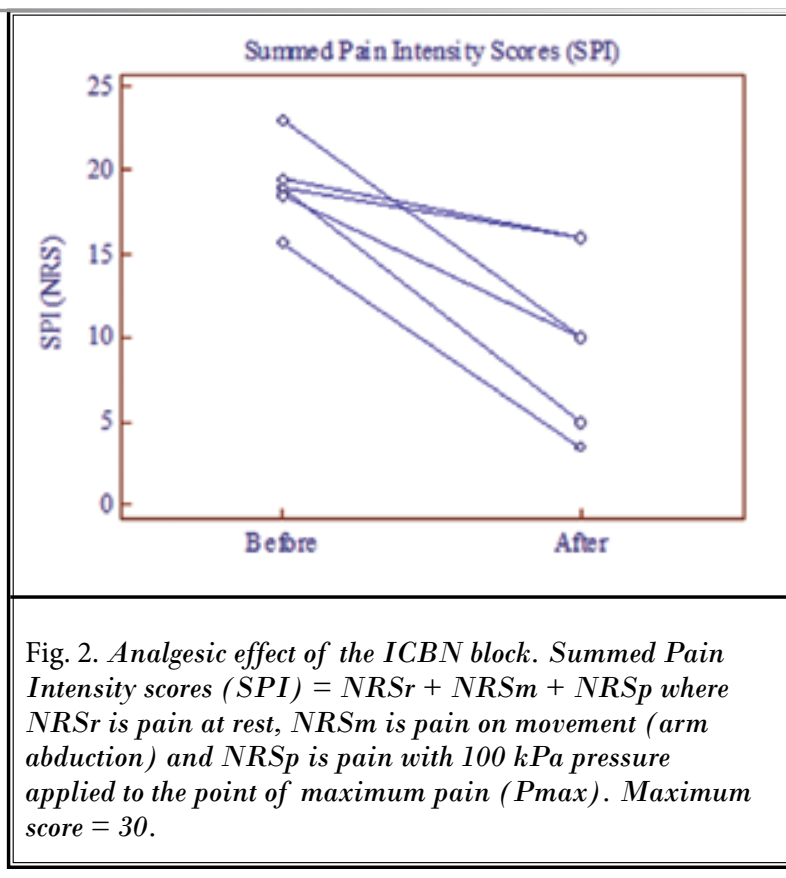

ence in SPI as the primary outcome. A priori analysis was performed using a 2-tailed t-test where the mean difference was 9, standard deviation 4.4, power (1- $\beta$ ) $=0.95$, and significance level $(\alpha)=0.05$. The number of patients for this trial would be 6 in each treatment group.

\section{Discussion}

This first pilot study demonstrates that local anesthetic blockade of the ICBN is possible using ultrasound guidance and the block can potentially provide pain relief in patients with PPBCS.

Ultrasound guided regional anesthesia (UGRA) has revolutionized the use of peripheral nerve blockade during the perioperative period and is proving to be 


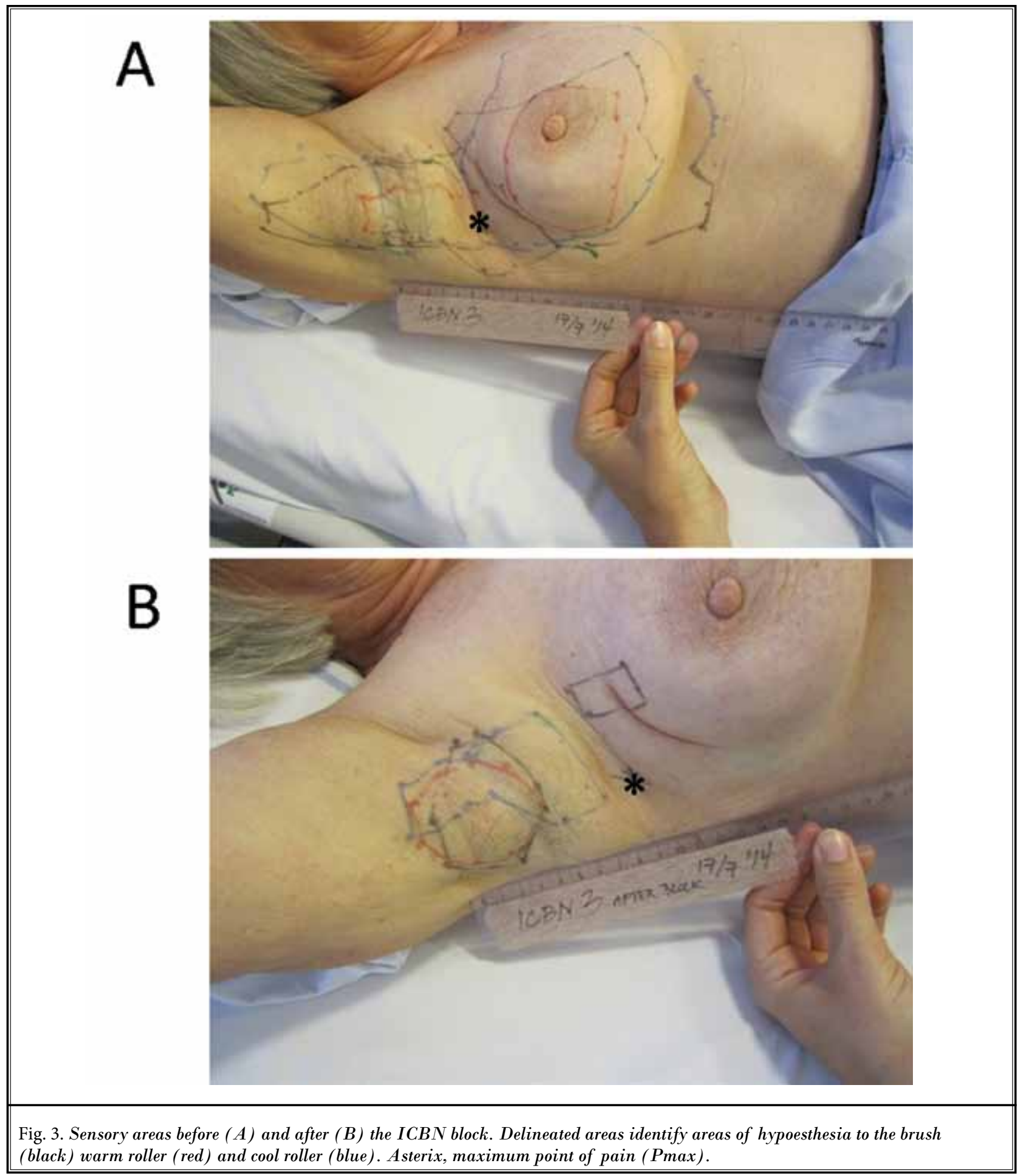

beneficial over traditional methods of blockade (13). However, there is a dearth of evidence for its use in pain medicine despite potential benefits such as reduced radiation exposure (14). Instead, in chronic pain medi- cine, UGRA is used for feasibility studies in cadavers or non-comparative patient models, which limits their usefulness clinically (13). In contrast, the present study is immediately clinically relevant as we have described 

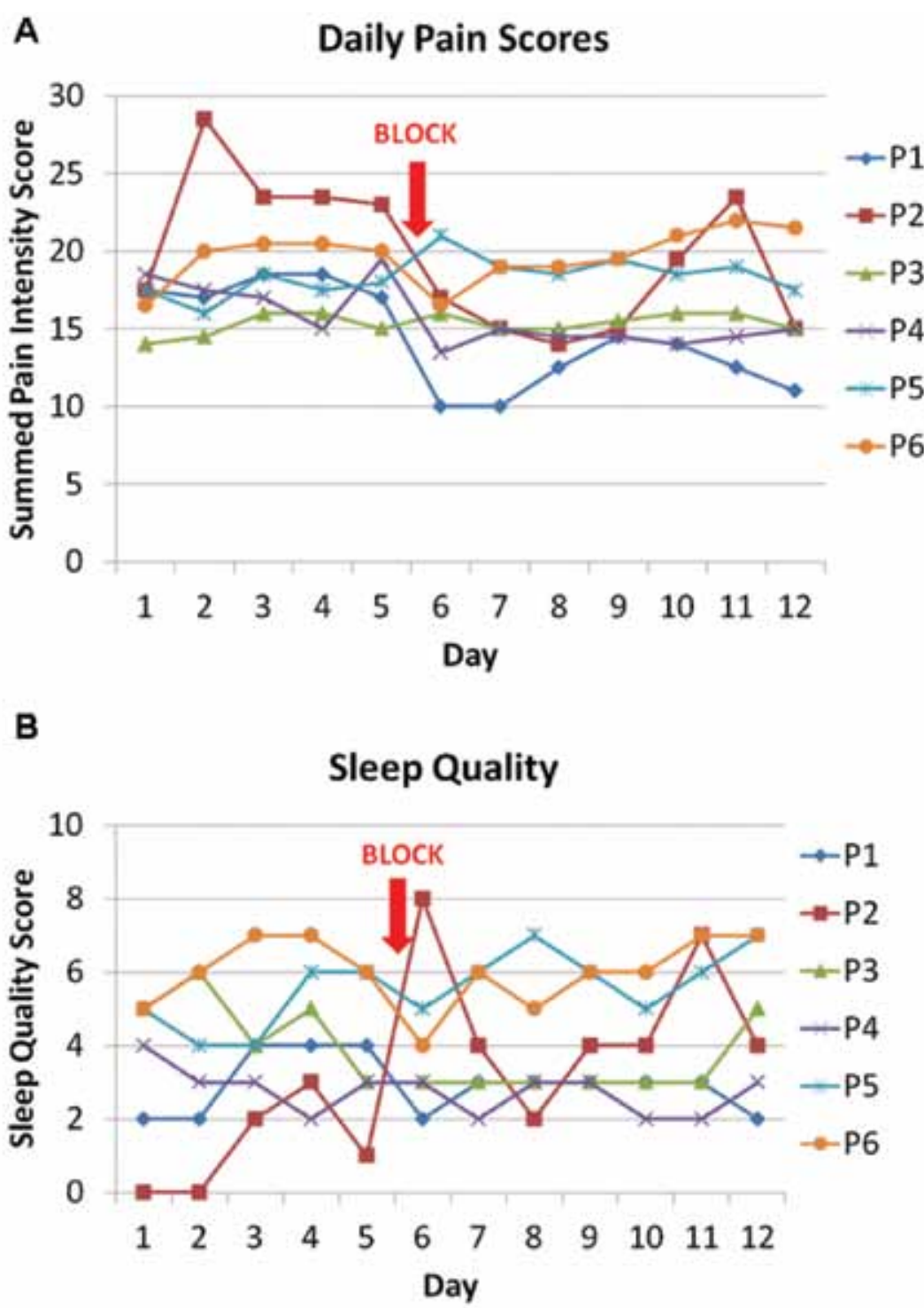

Fig. 4. Pain diary results. A: The patients noted their pain scores using the NRS twice a day in 3 predetermined states: at rest (supine), on movement $\left(90^{\circ}\right.$ arm abduction) and with manual pressure on the maximum point of pain (Pmax). B: Sleep quality was assessed daily using a numerical scoring system from 0 to 10 where $0=$ pain had no effect on sleep, $10=$ pain totally affected sleep.

and performed the ICBN block in the patient group that it is meant for. Ultrasonography was used to determine the sonoanatomy of the second intercostal space where the ICBN exits the thoracic cage in $100 \%$ of cases (5). However, there are a number of anatomical variations that may make the contribution of the T2 branch less significant and thus we cannot rule out the likelihood of a failed block (5). On the other hand, it could be an advantage that we performed a fascial plane block as this may be able to target some of the other branches, such as T1 or T3 that contribute to the ICBN. We also found that the long thoracic nerve, which lies in close proximity to the ICBN, was unaffected as none of the patients developed winged scapulae after the block. Ultrasonography described this anatomy in patients with a BMI between 20 and $38 \mathrm{~kg} \mathrm{~m}^{-2}$ consistently, but 
in a patient with a BMI $49 \mathrm{~kg} \mathrm{~m}^{-2}$, we were unable to visualize the second intercostal space. Therefore, when recruiting patients for the second part of the study we excluded patients with a BMI $\geq 40 \mathrm{~kg} \mathrm{~m}^{-2}$. Further studies using a curvilinear probe are needed to assess the ICBN block in morbidly obese patients with PPBCS.

The problem of PPBCS is well described $(2,15)$ and most evidence points towards the ICBN as one of the many culprits $(1,2)$. Yet it is surprising that the ICBN block has never been attempted. A recent review of neural blockade in PPBCS discussed the potential of ICBN blockade for diagnosis and treatment of PPBCS (7). In the review there were several small studies that used local anesthetic blockade of the intercostal nerves and paravertebral nerves for identification prior to surgical excision or neurolytic treatments. In our study 3 out of 6 patients had a reduction in pain of $>50 \%$ after the ICBN block, a criteria used in other studies for identification of suitability for further treatment (7). Consequently, a randomized placebo controlled study is needed to examine the possibility of using the ICBN block as a diagnostic tool and this pilot study was the first step towards that.

All of our patients had reduced pain scores after the local anesthetic (LA) block but 4 out of the 6 had a drop of $\geq 8.5$ points on NRS, which is clinically meaningful. These 4 patients were thrilled to have a moment of pain relief after suffering years of pain and trying all forms of analgesics that provided inadequate analgesia combined with terrible side effects. If we look at the pain scores in more detail, the significant reductions in pain were seen in pain on movement and with minimal (100 kPa) pressure. Clinically, these effects are also significant as patients daily functions are restricted by pain (12) and the reduction in pain on movement would result in a better quality of life. In addition, the reduction in pain led to an improved range of movement for one patient from a shoulder abduction of $40^{\circ}$ before vs. $100^{\circ}$ after the block (all the other patients had unrestricted shoulder movement to begin with). As previously mentioned, all patients experienced a reduction in pressure-evoked pain, and this was corroborated by the observed increase in PPTs. The LA was injected at a separate site to where the pressure algometry measurements were carried out so it cannot be a cushioning effect of the LA itself but the effect on the ICBN nerve that is causing the reduction in pain.

Interestingly, the 4 patients that had the largest reduction in pain demonstrated shrinkage of their areas of hypoesthesia. It is well known that LA causes numbness or loss of sensation in the blocked area, yet these 4 patients could feel light touch, warmth, and cold (30 minutes) after the block; whereas before the block they could barely feel these 3 modalities in the same area. In addition, 2 patients demonstrated a mirroring effect (16) exhibiting hypoesthesia areas on the opposite side to the operated (painful) side. The hypoesthesia areas also diminished in size on the mirroring side after the ICBN block in both patients, suggesting that these effects may be due to modification of central sensitization.

Two previous studies have demonstrated an analgesic effect after LA blockade of peripheral nerves yet had differing results when measuring sensation $(17,18)$. One study of peripheral nerve blockade in patients with peripheral neuropathy of the foot demonstrated an analgesic effect from LA blockade on spontaneous and evoked pain responses but markedly reduced sensation (17). The other study investigated the effect of LA blockade of the arm in patients with complex regional pain syndrome and observed a reduction in spontaneous pain and mechano- and cold-allodynia yet sensation was intact (18). Although the effects on sensation were different, both sets of authors suggest that peripheral afferent input is important in maintaining pain after peripheral nerve injury, which could be a valid explanation for our observations.

Results from the pain diaries suggest that the analgesic effect may outlast the conduction block of the LA as 3 patients experienced prolonged pain relief for more than 24 hours and after a week their pain scores have not returned to their baseline. Furthermore, 2 of these 3 patients reported functional improvement after a week as a direct result of their reduced pain. Interestingly, one patient reported 3 months of pain relief from the block and 2 patients have requested a repeat block. This effect has been seen in trigger point injections in PPBCS patients (19), although these investigators performed multiple injections precluding interpretation of the effect of the first injection. Indeed, the problem with the vast majority of LA nerve block studies is that investigators compare patients that have had variable numbers of blocks over variable time periods $(8,20)$.

The main limitation of our pilot study is the small sample size but this area of research is somewhat uncharted territory and the ICBN block is an invasive procedure. Therefore it would be unethical to enroll a large number of patients in an invasive, time consuming (for the patients) RCT where the effects were com- 
pletely unknown. In fact, we chose a suitable number of patients, as the sample size calculation based on our results have demonstrated. The premise of this study was to assess the feasibility of a RCT and our results suggest that a RCT is needed to ascertain the role of ICBN blockade in PPBCS.

\section{Author Contribution}

Drs. Wijayasinghe and Andersen and RN Duriaud had full access to all the data in the study and take responsibility for the integrity of the data and the accuracy of the data analysis. Drs. Wijayasinghe, Andersen, and Kehlet designed the study protocol. Dr Wijayasing- he managed the literature searches and summaries of previous related work and wrote the first draft of the manuscript. Drs. Wijayasinghe, Andersen, and Kehlet, and RN Duriaud provided revision for intellectual content and final approval of the manuscript.

\section{Role of Funders}

The funders of this work had no role in the design and conduct of the study or the collection, management, analysis, and interpretation of the data. The funders also did not have a role in the preparation or review of the manuscript or the decison to submit.

\section{References}

1. Andersen KG, Kehlet H. Persistent pain after breast cancer treatment: A critical review of risk factors and strategies for prevention. J Pain 2011; 12:725-746.

2. Schreiber KL, Kehlet H, Belfer I, Edwards RR. Predicting, preventing and managing persistent pain after breast cancer surgery: The importance of psychosocial factors. Pain Manag 2014; 4:445-459.

3. Mejdahl MK, Andersen KG, Gartner R, Kroman N, Kehlet H. Persistent pain and sensory disturbances after treatment for breast cancer: Six year nationwide follow-up study. BMJ 2013; 346:f1865.

4. Warrier S, Hwang S, Koh CE, Shepherd $H$, Mak $C$, Carmalt $H$, Solomon M. Preservation or division of the intercostobrachial nerve in axillary dissection for breast cancer: Meta-analysis of randomised controlled trials. Breast 2014; 23:310-316.

5. Loukas M, Hullett J, Louis RG Jr, Holdman S, Holdman D. The gross anatomy of the extrathoracic course of the intercostobrachial nerve. Clin Anat 2006; 19:106-111.

6. Andersen KG, Jensen MB, TvedeskovTF, Kehlet H, Gartner R, Kroman N. Persistent pain, sensory disturbances and functional impairment after immediate or delayed axillary lymph node dissection. Eur J Surg Oncol 2013; 39:31-35.

7. Wijayasinghe N, Andersen K G, Kehlet $\mathrm{H}$. Neural blockade for persistent pain after breast cancer surgery. Reg Anesth Pain Med 2014; 39:272-278.

8. Vlassakov KV, Narang S, Kissin I. Local anesthetic blockade of peripheral nerves for treatment of neuralgias: Systematic analysis. Anesth Analg 2011; 112:1487-1493.

9. Andersen KG, Aasvang EK, Kroman N Kehlet $\mathrm{H}$. Intercostobrachial nerve handling and pain after axillary lymph node dissection for breast cancer. Acta Anaesthesiol Scand 2014; 58:1240-1248.

10. Andersen KG, Kehlet H, Aasvang, EK. Test-retest agreement and reliability of quantitative sensory testing 1 year after breast cancer surgery. Clin J Pain 2015; 31:393-403

11. Bischoff JM, Koscielniak-Nielsen ZJ, Kehlet $H$, Werner MU. Ultrasoundguided ilioinguinal/iliohypogastric nerve blocks for persistent inguinal postherniorrhaphy pain: A randomized, doubleblind, placebo-controlled, crossover trial. Anesth Analg 2012; 114:1323-1329.

12. Andersen KG, Christensen KB, Kehlet $H$, Bidstup PE. The effect of pain on physical functioning after breast cancer treatment: Development and validation of an assessment tool. Clin J Pain 2015; 31:794-802.

13. Neal JM, Brull R, Chan VW, Grant SA, Horn JL, Liu SS, McCartney CJ, Narouze SN, Perlas A, Salinas FV, Sites BD, Tsui $B C$. The ASRA evidence-based medicine assessment of ultrasound-guided regional anesthesia and pain medicine: Executive summary. Reg Anesth Pain Med 2010; 35:S1-S9.

14. Gofeld M. Ultrasonography in pain medicine: A critical review. Pain Pract 2008; 8:226-240.
15. Bruce J, Thornton AJ, Powell R, Johnston M, Wells M, Heys SD, Thompson AM, Cairns SW, Chambers WA, Scott NW. Psychological, surgical, and sociodemographic predictors of pain outcomes after breast cancer surgery: A population-based cohort study. Pain 2014; 155:232-243.

16. Werner MU, Ringsted TK, Kehlet H, and Wildgaard K. Sensory testing in patients with postthoracotomy pain syndrome: Part 1: Mirror-image sensory dysfunction. Clin J Pain 2013; 29:775-783.

17. Haroutounian S, Nikolajsen L, Bendtsen TF, Finnerup NB, Kristensen AD, Hasselstrom JB, Jensen TS. Primary afferent input critical for maintaining spontaneous pain in peripheral neuropathy. Pain 2014; 155:1272-1279.

18. Gracely RH, Lynch SA, Bennett GJ. Painful neuropathy: altered central processing maintained dynamically by peripheral input. Pain, 51:175-194.

19. Shin HJ, Shin JC, Kim WS, Chang WH, Lee SC. Application of ultrasound-guided trigger point injection for myofascial trigger points in the subscapularis and pectoralis muscles to post-mastectomy patients: A pilot study. Yonsei Med ] 2014; 55:792-799.

20. Arner S, Lindblom U, Meyerson BA, Molander $C$. Prolonged relief of neuralgia after regional anesthetic blocks. A call for further experimental and systematic clinical studies. Pain 1990; 43:287-297. 
